MATHEMATICS OF COMPUTATION

Volume 68, Number 225, January 1999, Pages 351-369

S 0025-5718(99)00995-3

\title{
ANALYSIS OF PSLQ, AN INTEGER RELATION FINDING ALGORITHM
}

\author{
HELAMAN R. P. FERGUSON, DAVID H. BAILEY, AND STEVE ARNO
}

\begin{abstract}
Let $\mathbb{K}$ be either the real, complex, or quaternion number system and let $\mathbb{O}(\mathbb{K})$ be the corresponding integers. Let $x=\left(x_{1}, \ldots, x_{n}\right)$ be a vector in $\mathbb{K}^{n}$. The vector $x$ has an integer relation if there exists a vector $m=$ $\left(m_{1}, \ldots, m_{n}\right) \in \mathbb{O}(\mathbb{K})^{n}, m \neq 0$, such that $m_{1} x_{1}+m_{2} x_{2}+\ldots+m_{n} x_{n}=0$. In this paper we define the parameterized integer relation construction algorithm $\operatorname{PSLQ}(\tau)$, where the parameter $\tau$ can be freely chosen in a certain interval.

Beginning with an arbitrary vector $x=\left(x_{1}, \ldots, x_{n}\right) \in \mathbb{K}^{n}$, iterations of $\operatorname{PSLQ}(\tau)$ will produce lower bounds on the norm of any possible relation for $x$. Thus $\operatorname{PSLQ}(\tau)$ can be used to prove that there are no relations for $x$ of norm less than a given size. Let $M_{x}$ be the smallest norm of any relation for $x$. For the real and complex case and each fixed parameter $\tau$ in a certain interval, we prove that $\operatorname{PSLQ}(\tau)$ constructs a relation in less than $O\left(n^{3}+n^{2} \log M_{x}\right)$ iterations.
\end{abstract}

\section{InTRODUCTION}

Let $\mathbb{K}$ be either the real, complex or quaternion number system and let $\mathbb{O}(\mathbb{K})$ be the corresponding system of integers (i.e., ordinary integers, Gaussian integers, or Hamiltonian integers, respectively). Let $x=\left(x_{1}, \ldots, x_{n}\right)$ be a vector in $\mathbb{K}^{n}$. The vector $x$ has an integer relation if there exists a vector $m=\left(m_{1}, \ldots, m_{n}\right) \in \mathbb{O}(\mathbb{K})^{n}$, $m \neq 0$, such that $m_{1} x_{1}+m_{2} x_{2}+\ldots+m_{n} x_{n}=0$.

In this paper we define the parameterized integer relation construction algorithm $\operatorname{PSLQ}(\tau)$, which, compared with other integer relation algorithms in the literature, features superior performance and excellent numerical stability. The parameter $\tau$ can be freely chosen in the interval $1<\tau<\rho$, where $\rho$ is 2 or $\sqrt{2}$ depending on whether $\mathbb{K}$ is the reals or complexes, respectively; if $\mathbb{K}$ is the quaternions take $\tau$ and $\rho$ to be 1 . We analyze $\operatorname{PSLQ}(\tau)$ for these three number systems. We describe in detail some efficient Fortran multiprecision computer implementations of PSLQ $(\tau)$.

Beginning with an arbitrary vector $x=\left(x_{1}, \ldots, x_{n}\right) \in \mathbb{K}^{n}$, a finite number of iterations of $\operatorname{PSLQ}(\tau)$ will produce lower bounds on the (Frobenius) norm of any possible relation for $x$. The computation of such a lower bound constitutes a proof that $x$ has no integer relations whatsoever of norm less than this lower bound. Any finite computation done with PSLQ or any other presently known relation finding algorithm can only prove that no small relation exists. Such an algorithm can construct an alleged relation based on inputs given to finite precision, but the proof that this alleged relation is a true relation for the real numbers is a separate matter.

Received by the editor April 12, 1996 and, in revised form, June 9, 1997.

1991 Mathematics Subject Classification. Primary 11A05, 11Y16, 68-04.

Key words and phrases. Euclidean algorithm, integer relation finding algorithm, Gaussian integer, Hamiltonian integer, polynomial time. 
Let $M_{x}$ be the smallest norm of a relation for $x$. Define $\gamma$ by the equation $\tau=1 / \sqrt{1 / \rho^{2}+1 / \gamma^{2}}$, where $\rho=2$ for the real number field and $\rho=\sqrt{2}$ for the complex number field, and $\tau$ is as above. For each fixed parameter $\tau$ in the interval $1<\tau<\rho$, we prove in the real and complex case that $\operatorname{PSLQ}(\tau)$ constructs a relation in less than $\left(\begin{array}{l}n \\ 2\end{array}\right) \log _{\tau}\left(\gamma^{n-1} M_{x}\right)$ iterations. This shows that $\operatorname{PSLQ}(\tau)$ is 'polynomial time' in the dimension and the number of bits of a smallest integer relation. Different choices of $\tau$ or $\gamma$ lead to different time and space requirements for the algorithm.

For dimension $n=2$ we prove that $\operatorname{PSLQ}(\tau)$ will construct a relation of smallest norm $M_{x}$. We give examples in dimension $n=3$, for some $\tau$, for which PSLQ $(\tau)$ does not construct a relation of smallest norm $M_{x}$. However for any dimension $n \geq 2$, we do prove that any relation constructed by $\operatorname{PSLQ}(\tau)$ has norm less than or equal to $\gamma^{n-2} M_{x}$.

The 'polynomial time' and 'small norm' proofs given here are straightforward generalizations to the parameter $\tau$ and to the complex numbers of the original 'polynomial time' proofs which appear in Lagarias et al., [23]. We show, however, that the algorithm of [23] is distinct from any of these PSLQ $(\tau)$ algorithms.

$\operatorname{PSLQ}(\tau)$ was introduced by the authors [2] in 1991. PS refers to partial sums of squares, LQ to a lower trapezoidal orthogonal decomposition, and $(\tau)$ is a parameter defined as above. Since PSLQ $(\tau)$ was introduced it has been used to discover numerous previously unknown identities among real numbers. One example is

$$
\begin{aligned}
& \sum_{k=1}^{\infty}\left(1-\frac{1}{2}+\cdots+\frac{(-1)^{k+1}}{k}\right)^{2}(k+1)^{-3} \\
= & 4 L_{5}(1 / 2)-\frac{1}{30} \ln ^{5}(2)-\frac{17}{32} \zeta(5)-\frac{11}{720} \pi^{4} \ln (2) \\
& +\frac{7}{4} \zeta(3) \ln ^{2}(2)+\frac{1}{18} \pi^{2} \ln ^{3}(2)-\frac{3}{24} \pi^{2} \zeta(3),
\end{aligned}
$$

where $L_{n}(x)$ denotes the polylogarithm function $\sum_{k} x^{k} k^{-n}$. See [3] for details. Another example is the following formula for $\pi$ :

$$
\pi=\sum_{i=0}^{\infty} \frac{1}{16^{i}}\left(\frac{4}{8 i+1}-\frac{2}{8 i+4}-\frac{1}{8 i+5}-\frac{1}{8 i+6}\right) .
$$

This remarkable series permits one to rapidly compute individual digits from the hexadecimal expansion of $\pi$. See [4] for details. It was found by applying PSLQ $(\tau)$ to the vector $X=\left(X_{1}, X_{2}, \cdots, X_{8}, \pi\right)$ where $X_{j}=\sum_{k \geq 0} 1 /\left(16^{k}(8 k+j)\right)$. The smallest relation known,

$$
(4,0,0,-2,-1,-1,0,0,-1)
$$

yields the above 'base 16' formula for $\pi$. A next smallest relation known,

$$
(0,8,4,4,0,0,-1,0,-2)
$$

was subsequently discovered by Ferguson, and this relation yields a similar 'base 16 ' formula for $\pi$. Together these two integral lattice relation vectors generate a two-dimensional lattice of relations of this 'base 16 ' type. It is conjectured that there are no further such relations outside this lattice. Note that

$$
(-8,8,4,8,2,2,-1,0,0)
$$

is in this lattice, so evidently $X_{7}$ is integrally dependent upon $X_{1}, \ldots, X_{6}$. 
Of course, a numerical discovery of a relation using PSLQ $(\tau)$ does not constitute a rigorous proof of the relation. However, in the wake of this numerical evidence, proofs have subsequently been found for many of these relations, including the above formula for $\pi$. See [3] and [4] for details.

In the theoretical proofs in Sections 2, 3, 4, and 5, we will assume exact arithmetic over the real numbers augmented by comparisons over the reals and the nearest integer function.

\section{LOWER BOUNDS ON INTEGER RELATIONS}

If $\mathbb{K}$ is the complex number field, then $z^{\star}$ denotes the complex conjugate of $z$, i.e. if $z=x+i y$, then $z^{\star}=x-i y .|\cdot|$ denotes the complex absolute value, i.e. $|z|^{2}=z^{\star} z=z z^{\star}=x^{2}+y^{2}$. If $A$ is a matrix or vector, then $A^{\star}$ is the conjugate transpose of $A$. A unit in the complex number field is any element $z$ such that $|z|=1$. For real $z$, the conjugate operation is null, and $|z|$ is the usual absolute value.

Similarly, if $\mathbb{K}$ is the quaternion number system, then $z^{\star}$ denotes the quaternion conjugate of $z$, i.e. if $z=x+y i+u j+v k$, then $z^{\star}=x-y i-u j-v k$. The quaternion absolute value or norm is similarly defined, so that $|z|^{2}=z z^{\star}=z^{\star} z=$ $x^{2}+y^{2}+u^{2}+v^{2}$. Units and conjugates of matrices are defined analogously.

If $\mathbb{K}$ is any of the above three number systems, two vectors $x, y \in \mathbb{K}^{n}$ are said to be orthogonal if $x y^{\star}=0$. Let $|A|=\left(\operatorname{tr}\left(A^{\star} A\right)\right)^{1 / 2}$ denote the Frobenius norm of the matrix $A$, i.e., $|A|=\left(\sum a_{i, j}^{\star} a_{i, j}\right)^{1 / 2}$. An $n \times n$ matrix $A$ is unitary if $A^{\star} A=A A^{\star}=I_{n} . U(n, \mathbb{K})$ denotes the group of unitary matrices over $\mathbb{K}$. An $n \times n$ matrix $A$ is unimodular if $\operatorname{det} A$ is a unit. $G L(n, \mathbb{O}(\mathbb{K}))$ is the group of unimodular matrices with entries in the integers $\mathbb{O}(\mathbb{K})$.

Definition $1\left(M_{x}\right)$. Assume $x=\left(x_{1}, \ldots, x_{n}\right) \in \mathbb{K}^{n}$ has norm $|x|=1$. Define $x^{\perp}$ to be the set of all vectors in $\mathbb{K}^{n}$ orthogonal to $x$. Let $\mathbb{O}(\mathbb{K})^{n} \cap x^{\perp}$ be the discrete lattice of integral relations for $x$. Define $M_{x}>0$ to be the smallest norm of any relation for $x$ in this lattice.

Definition $2\left(H_{x}\right)$. Assume $x=\left(x_{1}, \ldots, x_{n}\right) \in \mathbb{K}^{n}$ has norm $|x|=1$. Furthermore, suppose that no coordinate entry of $x$ is zero, i.e., $x_{j} \neq 0$ for $1 \leq j \leq n$ (otherwise $x$ has an immediate and obvious integral relation). For $1 \leq j \leq n$ define the partial sums

$$
s_{j}^{2}=\sum_{j \leq k \leq n} x_{k} x_{k}^{\star} .
$$

Given such a unit vector $x$, define the $n \times(n-1)$ lower trapezoidal matrix $H_{x}=\left(h_{i, j}\right)$ by

$$
h_{i, j}= \begin{cases}0 & \text { if } 1 \leq i<j \leq n-1, \\ s_{i+1} / s_{i} & \text { if } 1 \leq i=j \leq n-1, \\ -x_{i}^{\star} x_{j} /\left(s_{j} s_{j+1}\right) & \text { if } 1 \leq j<i \leq n .\end{cases}
$$

Note that $h_{i, j}$ is scale invariant.

Lemma 1. Let $H_{x}$ be the lower trapezoidal matrix defined above. Then:

(i) $H_{x}^{\star} H_{x}=I_{n-1}$, i.e., the columns of $H_{x}$ are orthogonal,

(ii) $\left|H_{x}\right|=\sqrt{n-1}$,

(iii) $x H_{x}=0$. 
Proof. The columns can be proven orthogonal by considering the cases $i=j$ and $i<j$ separately. When $i=j$ the inner product is

$$
\begin{gathered}
\frac{s_{i+1}^{2}}{s_{i}^{2}}+\sum_{i<k \leq n} \frac{x_{i} x_{i}^{\star} x_{k} x_{k}^{\star}}{s_{i}^{2} s_{i+1}^{2}}=\frac{s_{i+1}^{2}}{s_{i}^{2}}+\frac{x_{i} x_{i}^{\star}}{s_{i}^{2} s_{i+1}^{2}} \sum_{i<k \leq n} x_{k} x_{k}^{\star} \\
=\frac{s_{i}^{2}-x_{i} x_{i}^{\star}}{s_{i}^{2}}+\frac{x_{i} x_{i}^{\star}}{s_{i}^{2}}=1 .
\end{gathered}
$$

When $i<j$ the inner product is

$$
\begin{gathered}
-\frac{s_{j+1} x_{i}^{\star} x_{j}}{s_{j} s_{i} s_{i+1}}+\sum_{j<k \leq n} \frac{x_{i}^{\star} x_{j} x_{k} x_{k}^{\star}}{s_{i} s_{i+1} s_{j} s_{j+1}} \\
=-\frac{s_{j+1} x_{i}^{\star} x_{j}}{s_{j} s_{i} s_{i+1}}+\frac{x_{i}^{\star} x_{j}}{s_{i} s_{i+1} s_{j} s_{j+1}} \sum_{j<k \leq n} x_{k} x_{k}^{\star}=0 .
\end{gathered}
$$

Item (i) shows that $H_{x}^{\star} H_{x}=I_{n-1}$, which has trace $n-1$, so $\left|H_{x}\right|=\sqrt{n-1}$. To prove (iii), fix $1 \leq j \leq n-1$; then

$$
\sum_{1 \leq k \leq n} x_{k} h_{k, j}=\frac{x_{j} s_{j+1}}{s_{j}}-\sum_{j<k \leq n} \frac{x_{k} x_{k}^{\star} x_{j}}{s_{j} s_{j+1}}=\frac{x_{j} s_{j+1}}{s_{j}}-\frac{x_{j} s_{j+1}^{2}}{s_{j} s_{j+1}}=0 .
$$

Lemma 2. For a unit vector $x \in \mathbb{K}^{n}$ define $P_{x}=H_{x} H_{x}^{\star}$. Then $P_{x}$ satisfies:

(i) $P_{x}^{\star}=P_{x}$,

(ii) $P_{x}=I_{n}-x^{\star} x$,

(iii) $P_{x}^{2}=P_{x}$

(iv) $\left|P_{x}\right|=\sqrt{n-1}$,

(v) $P_{x} z^{\star}=z^{\star}$ for any $z \in x^{\perp}$,

(vi) $P_{x} m^{\star}=m^{\star}$ for any relation $m \in \mathbb{O}(\mathbb{K})^{n}$ for $x$.

Proof. Item (i) follows from $H_{x} H_{x}^{\star}=\left(H_{x} H_{x}^{\star}\right)^{\star}$. To prove (ii) note that, from Lemma 1 (iii), $H_{x}$ is an $n \times(n-1)$ rank $n-1$ matrix whose columns transposed form an orthonormal basis for $x^{\perp}$. Defining $U=\left(H_{x} \mid x^{\star}\right)$, an $n \times n$ unitary matrix, we have $U U^{\star}=H_{x} H_{x}^{\star}+x^{\star} x=I_{n}$. To prove (iii), note that

$$
P_{x}^{2}=\left(I_{n}-x^{\star} x\right)^{2}=I_{n}^{2}-2 I_{n} x^{\star} x+x^{\star}\left(x x^{\star}\right) x=P_{x} .
$$

To prove (iv), note that $\left|P_{x}\right|^{2}=\operatorname{tr}\left(P_{x}^{\star} P_{x}\right)=\operatorname{tr} P_{x}=\operatorname{tr} H_{x}^{\star} H_{x}=n-1$. Item (vi) follows from (v), which follows from (ii) and the associativity $\left(x^{\star} x\right) z^{\star}=x^{\star}\left(x z^{\star}\right)$.

Theorem 1. Let $x \neq 0 \in \mathbb{K}^{n}$. Suppose that for any relation $m$ of $x$ and for any matrix $A \in G L(n, \mathbb{O}(\mathbb{K}))$ there exists a unitary matrix $Q \in U(n-1)$ such that $H=A H_{x} Q$ is lower trapezoidal and all of the diagonal elements of $H$ satisfy $h_{j, j} \neq 0$. Then

$$
\frac{1}{\max _{1 \leq j \leq n-1}\left|h_{j, j}\right|}=\min _{1 \leq j \leq n-1} \frac{1}{\left|h_{j, j}\right|} \leq|m| .
$$


Proof. Let $m$ be any relation for $x$. By the hypothesis, there exists a unitary matrix $Q \in U(n-1)$ such that $H=A H_{x} Q$ is lower trapezoidal (this is equivalent to QR factorization). There is an $n \times n-1$ matrix $T$ with diagonal ones and an $n-1 \times n-1$ diagonal matrix $D$ where $H=T D$ with diagonal entries $h_{j, j} \neq 0,1 \leq j \leq n-1$, from the hypothesis. On the other hand, $A P_{x}=H Q^{\star} H_{x}^{\star}$, from the definition of $P_{x}$ in Lemma 2. The equation $A P_{x}=T D Q^{\star} H_{x}^{\star}$ gives a decomposition of $A P_{x}$ into the product of a lower trapezoidal matrix $T$ with diagonal 1's, an invertible diagonal matrix $D$ with diagonal $h$ 's, and an $n-1 \times n$ matrix $Q^{\star} H_{x}^{\star}$ with orthonormal rows, since $Q^{\star} H_{x}^{\star} H_{x} Q=Q^{\star} I_{n-1} Q=I_{n-1}$ by Lemma 1 . So the norm of the $j$-th row of $D Q^{\star} H_{x}^{\star}$ is $\left|h_{j, j}\right|$.

From Lemma 2, part (vi), $m^{\star}=P_{x} m^{\star}$, so that $A m^{\star}=A P_{x} m^{\star}$. From the above decomposition of $A P_{x}=T D Q^{\star} H_{x}^{\star}$, we have $A m^{\star}=A P_{x} m^{\star}=T D\left(Q^{\star} H_{x}^{\star}\right) m^{\star}$. Let $Q_{H, j}$ be the $j$-th row of $Q^{\star} H_{x}^{\star}$ and let $A_{j}$ be the $j$-th row of $A$. Then

$$
A_{j} m^{\star}=h_{j, j} Q_{H, j} m^{\star}+\sum_{k<j} t_{j, k} h_{k, k} Q_{H, k} m^{\star} .
$$

Since $A$ is invertible, $A m^{\star} \neq 0$. Let $j$ be the least $j$ for which $A_{j} m^{\star} \neq 0$, so that $A_{k} m^{\star}=0$ for $k<j$. Then the $k<j$ rows of $T D Q^{\star} H_{x}^{\star} m^{\star}$ are zero, and since $T$ is lower trapezoidal by recursion, the $k$-th rows of $Q^{\star} H_{x}^{\star} m^{\star}$ are also zero. With this least choice of $j$, then, $A_{j} m^{\star}=h_{j, j} Q_{j} m^{\star}$. Therefore, from $A \in G L(n, \mathbb{O}(\mathbb{K}))$,

$$
1 \leq\left|A_{j} m^{\star}\right| \leq\left|h_{j, j} Q_{H, j} m^{\star}\right| \leq\left|h_{j, j}\right|\left|m^{\star}\right|,
$$

because $Q_{H, j}$ is a unit vector.

Comment on Theorem 1. Theorem 1 suggests a strategy to construct a relation finding algorithm: Find a way to reduce the norm of the matrix $H_{x}$ by multiplication by some unimodular $A$ on the left. The inequality of Theorem 1 offers an increasing lower bound on the size of any possible relation. Theorem 1 can be used with any algorithm that produces any $G L(n, \mathbb{O}(\mathbb{K}))$ matrices. Any $G L(n, \mathbb{O}(\mathbb{K}))$ matrix $A$ whatsoever can be put into Theorem 1 .

Definition 3 (Hermite reduction). Let $H$ be a lower trapezoidal matrix, with $h_{i, j}=0$ if $j>i$ and $h_{j, j} \neq 0$. Define the matrix $D=\left(d_{i, j}\right) \in G L(n, \mathbb{O}(K))$ recursively as follows. For fixed $i$, decrement $j$ from $n$ to 1 , setting

$$
d_{i, j}= \begin{cases}0 & \text { if } i<j, \\ 1 & \text { if } i=j, \\ \operatorname{nint}\left(\left(-\sum_{j<k \leq i} d_{i, k} h_{k, j}\right) / h_{j, j}\right) & \text { if } j<i .\end{cases}
$$

We will say that $D H$ is the Hermite reduction of $H$ and that $D$ is the reducing matrix of $H$. The function nint denotes a nearest integer function, e.g., $\operatorname{nint}(t)=\lfloor t+1 / 2\rfloor$. This definition of nint can be extended to each coordinate for complex or quaternion arguments.

Definition 4 (Modified Hermite reduction). With the same notation as in Definition 3 , set $D=I_{n}$. For $i$ from 2 to $n$, and for $j$ from $i-1$ to 1 (step -1 ), set $q=\operatorname{nint}\left(h_{i, j} / h_{j, j}\right)$; then for $k$ from 1 to $j$ replace $h_{i, k}$ by $h_{i, k}-q h_{j, k}$, and for $k$ from 1 to $n$ replace $d_{i, k}$ by $d_{i, k}-q d_{j, k}$.

Lemma 3. For a lower triangular matrix $H$ with $h_{i, j}=0$ if $j>i$ and $h_{j, j} \neq 0$, Hermite reduction is equivalent to modified Hermite reduction. 
Comment. This variation can be found in [8] and later in [28]. This recursion replaces the input $H$ with $D H$ while developing the left multiplying reduction matrix $D$.

Lemma 4. There exists a constant $\rho_{\mathbb{K}}=\rho \geq 1$, with the property that the entries of the Hermite reduced matrix $H^{\prime}=\left(h_{i, j}^{\prime}\right)=D H$ satisfy the inequality

$$
\left|h_{k, i}^{\prime}\right| \leq\left|h_{i, i}^{\prime}\right| / \rho=\left|h_{i, i}\right| / \rho
$$

for all $k>i$. The constant $\rho=2$ for the real case, $\rho=\sqrt{2}$ for the complex case, and $\rho=1$ for the quaternion case.

Proof. This follows from the definitions of the nint function, Hermite reduction, and the fact that $|z-\operatorname{nint}(z)| \leq \sqrt{\operatorname{dim}_{\mathbb{R}} \mathbb{K}} / 2$ for $z \in \mathbb{K}$.

\section{Statement of the Algorithm PSLQ $(\tau)$}

Definition 5 (The parameters $\gamma$ and $\tau$ ). Fix the real number $\gamma>2 / \sqrt{3}$ or $\gamma>\sqrt{2}$ or $\gamma=\infty$ for the real, complex, and quaternion cases respectively. In terms of this $\gamma$, define the real number $\tau$ by

$$
1 / \tau^{2}=1 / \rho^{2}+1 / \gamma^{2}
$$

where $\rho$ is defined as in Lemma 4. For the proof of Theorem 2, we will require that $1<\tau$ and that $\tau \leq \rho$; clearly these conditions are satisfied in the real and complex cases. In the quaternion case $\tau=1$ and $\rho=1$.

For the proofs that follow assume $\mathbb{K}$ is real or complex, not quaternion. Note however that the statement of the algorithm is valid for the quaternions.

Initial conditions. Given the input unit vector $x \in \mathbb{K}^{n}$, set $H=H_{x}$, where $H_{x}$ is defined as above. Set the $n \times n$ matrices $A$ and $B$ equal to the identity $I_{n}$. Perform Hermite reduction on $H$, producing $D \in G L(n, \mathbb{O}(\mathbb{K}))$. Replace $x$ by $x D^{-1}, H$ by $D H, A$ by $D A, B$ by $B D^{-1}$.

\section{One four-step iteration.}

Step 1 : Exchange. Let $H=\left(h_{i, j}\right)$, where $h_{i, j}$ is the $i$-th row, $j$-th column entry of $H$. Let

$$
\alpha=h_{r, r}, \quad \beta=h_{r+1, r}, \quad \lambda=h_{r+1, r+1}, \quad \delta=\sqrt{\beta \beta^{\star}+\lambda \lambda^{\star}} .
$$

Choose an integer $r$ such that $\gamma^{r}\left|h_{r, r}\right| \geq \gamma^{i}\left|h_{i, i}\right|$ for all $1 \leq i \leq n-1$. Define the permutation matrix $R$ to be the identity matrix with the $r$ and $r+1$ rows exchanged. Replace $x$ by $x R, H$ by $R H, A$ by $R A$, and $B$ by $B R$.

Step 2 : Corner. At this point the updated matrix $H$ may not be lower trapezoidal since $\lambda$ may not be zero. If $r<n-1$, replace $H$ by $H Q$, where $Q$ is the unitary $n-1 \times n-1$ matrix $Q=\left(q_{i, j}\right) \in U(n-1, \mathbb{K})$ defined by

$$
q_{i, j}= \begin{cases}\beta^{\star} / \delta & \text { if } i=r, j=r, \\ -\lambda / \delta & \text { if } i=r, j=r+1, \\ \lambda^{\star} / \delta & \text { if } i=r+1, j=r, \\ \beta / \delta & \text { if } i=r+1, j=r+1, \\ 1 & \text { if } i=j \neq r \text { or } i=j \neq r+1, \\ 0 & \text { otherwise, }\end{cases}
$$

where the $\alpha, \beta, \lambda, \delta$ are defined in Step 1. If $r=n-1$ then $H$ is unchanged. 
Step 3 : Reduction. Perform Hermite reduction on $H$, producing $D \in G L(n, \mathbb{O}(\mathbb{K}))$. Replace $x$ by $x D^{-1}, H$ by $D H, A$ by $D A, B$ by $B D^{-1}$.

Step 4: Termination. Terminate the algorithm if $x_{j}=0$ for some $1 \leq j \leq n$ or if $h_{i, i}=0$ for some $1 \leq i \leq n-1$.

\section{Number of iterations of PSLQ $(\tau)$}

Let $H(k)=H, A$, and $B=A^{-1}$ be the result after exactly $k$ iterations of PSLQ. Let $\alpha=h_{r, r}(k)$ and $\beta=h_{r+1, r}(k)$. These definitions of $\alpha$ and $\beta$ are consonant with those of Step 2. Because $H$ is Hermite reduced in Step 3, from Lemma 4, $|\beta|<|\alpha| / \rho$. For $r<n-1$ set $\lambda=h_{r+1, r+1}(k)$ and define $t$ by $t=\sqrt{\beta \beta^{\star}+\lambda \lambda^{\star}} /|\alpha|$. From this definition of $t$ we have

$$
|\lambda| \leq|\alpha| t
$$

From the Step 1 Exchange, $0 \leq|\lambda| \leq|\alpha| / \gamma$. It follows that

$$
t=\sqrt{\beta \beta^{\star}+\lambda \lambda^{\star}} /|\alpha| \leq \sqrt{1 / \rho^{2}+1 / \gamma^{2}}=\tau,
$$

as in Definition 5 . For this proof we will require that $t<1<\tau$, clearly satisfied in the real and complex cases.

Lemma 5. If $h_{j, j}(k)=0$ for some $1 \leq j \leq n-1$ and no smaller $k$, then $j=n-1$ and a relation for $x$ must appear as a column of the matrix $B$.

Proof (Alyson Reeves). First we show that $h_{j, j}=0$ implies that $j=n-1$. Consider the matrix $H(k-1)$, the end result of the $k-1$-th iteration. By the hypothesis on $k$ we know that no diagonal elements in $H(k-1)$ are zero. In particular, for the $r$ about to be chosen in Step 1 of the $k$-th iteration, we know that $h_{r, r}(k-1) \neq 0$ and that $h_{r+1, r+1}(k-1) \neq 0$. Now, suppose the $r$ chosen in Step 1 is not $n-1$. Let

$$
\left(\begin{array}{ll}
\alpha & 0 \\
\beta & \lambda
\end{array}\right)
$$

be the submatrix of $H(k-1)$ consisting of the $r$ and $r+1$ rows of columns $r$ and $r+1$. After Step 1 has been performed this submatrix becomes

$$
\left(\begin{array}{ll}
\beta & \lambda \\
\alpha & 0
\end{array}\right)
$$

At Step 2, we post-multiply the matrix by the unitary sub-matrix of $Q$

$$
\left(\begin{array}{cc}
\beta^{\star} / \delta & -\lambda / \delta \\
\lambda^{\star} / \delta & \beta / \delta
\end{array}\right)
$$

where $\delta=\sqrt{\beta \beta^{\star}+\lambda \lambda^{\star}}$. The result is the matrix

$$
\left(\begin{array}{cc}
\delta & 0 \\
\alpha \beta^{\star} / \delta & -\alpha \lambda / \delta
\end{array}\right) \text {. }
$$

Since $\lambda$ and $\alpha$ are not zero (they were diagonal elements of $H(k-1)$ ), we know that $\delta$ and $-\alpha \lambda / \delta$, the two diagonal elements in the matrix, are also not zero. Note that since the rest of $Q$ is the identity matrix, none of the other diagonal elements is affected by the multiplication. Thus, at the end of Step 2, all diagonal elements are non-zero. Since Hermite reduction doesn't introduce any new zeros on the diagonal, the end result of the $k$-th iteration has all non-zero diagonal elements. But this contradicts the hypothesis on $k$ and our assumption that $r<n-1$ was 
false. Note that for $r=n-1$, in order to have $h_{n-1, n-1}(k)=0$, we must have $h_{n, n-1}(k-1)=0$ and $h_{n-1, n-1}(k-1) \neq 0$.

Next we show that a relation for $x$ must appear as a column of the matrix $B$. By Lemma $1, x H_{x}=0 . B A=I_{n}$ implies $0=x B A H_{x}=x B A H_{x} Q=x B H(k-1)$, where $Q$ is an appropriate unitary $n-1 \times n-1$ matrix. Let $z=x B$. The above gives

$$
(0, \ldots, 0)=x B H(k-1)=z H(k-1)=\left(\ldots, z_{n-1} h_{n-1, n-1}(k-1)\right) .
$$

Since $h_{n-1, n-1}(k-1) \neq 0$, then $z_{n-1}=0$. Hence the $n-1$-th column of $B$ is a relation for $x$.

Lemma 6. At any $k$-th iteration of the algorithm the diagonal entries of $H(k)$ satisfy the inequality $\left|h_{i, i}(k)\right| \leq 1$.

Proof. We follow the $\alpha, \beta, \lambda$ definitions of the proof of Lemma 5 and use induction. For $k=1$ the diagonal entries of $H(k)$ are those of $H_{x}$, and $s_{j+1} \leq s_{j} \leq 1$ gives the required inequality. Assume that the inequality also holds up to $k-1$. The diagonal entries of $H(k)$ are equal to those of $H(k-1)$ except for row $r$, where Step 1 Exchange occurs. When $r=n-1$, after the exchange, the $r$-th diagonal element is $\beta$. But $|\beta| \leq|\alpha| / \rho \leq 1$, because $\rho>1$ and $|\alpha| \leq 1$ by induction. When $r<n-1$, after the exchange the $r$-th diagonal element is $\delta$. But $|\delta|=|\alpha| t \leq 1$, since $t<1$ and $|\alpha| \leq 1$. The $r+1$-th diagonal element of $H$ is $-\alpha \lambda / \delta$ (as in the proof of Lemma 5), so that $|-\alpha \lambda / \delta|=|\lambda| / t \leq|\alpha|$, because $|\lambda|^{2}<|\lambda|^{2}+|\beta|^{2}$ and $|\lambda| \leq|\alpha| t$.

We show that every iteration of PSLQ causes a geometric monotonic increase in a certain function $\Pi(k)$ which is roughly the product of all the principal minors of the matrix $H(k)$. If a relation for $x$ exists, this product will be bounded above and below. Assume $x$ has some relation, and as usual let $M_{x}$ denote the norm of a smallest relation for $x$. We will need the following technical lemma in the proof of Lemma 9.

Lemma 7. Consider the quotient

$$
q(A, B, t)=\frac{\min \{B, t\} \cdot \min \{A, 1\}}{\min \{B, 1\} \cdot \min \{A, t\}} .
$$

Suppose that the four positive real numbers $A, B, 1, t$ satisfy the three inequalities

$$
A \geq B, \quad A \geq t, \quad 1 \geq t .
$$

Then,

$$
q(A, B, t) \geq 1 .
$$

Proof. Of the 16 possible choices in the min's, the inequality $A \geq t$ removes 8 , $A \geq B$ removes 2 , and $1 \geq t$ removes 1 , leaving 5 . These five are

$A \geq B \geq 1 \geq t$ with quotient $t / 1 \cdot 1 / t=1$

$A \geq 1 \geq B \geq t$ with quotient $t / B \cdot 1 / t \geq t / 1 \cdot 1 / t=1$,

$1 \geq A \geq B \geq t$ with quotient $t / B \cdot A / t=A / B \geq 1$,

$1 \geq A \geq t \geq B$ with quotient $B / B \cdot A / t=A / t \geq 1$,

$A \geq 1 \geq t \geq B$ with quotient $B / B \cdot 1 / t=1 / t \geq 1$.

Lemma 8. For $\alpha, \gamma, M_{x}$ as above,

$$
\gamma^{n-2} M_{x}|\alpha| \geq 1 .
$$


Proof. By the choice of $r$ in Step 1 Exchange, we have $\gamma^{r}|\alpha| \geq \gamma^{j}\left|h_{j, j}\right|$ for any $j, \quad 1 \leq j \leq n-1$, which implies

$$
\gamma^{n-1} /\left|h_{j, j}\right| \geq \gamma^{r} /\left|h_{j, j}\right| \geq \gamma^{j} /|\alpha| \geq \gamma^{1} /|\alpha|,
$$

for all $j$ including that $j_{o}$ for which $M_{x} \geq 1 /\left|h_{j_{o}, j_{o}}\right|$ from Theorem 1 . Thus $\gamma^{n-2} M_{x} \geq 1 /|\alpha|$ and $\gamma^{n-2} M_{x}|\alpha| \geq 1$

Definition 6 (The $\Pi$ function). Recall that $\tau=\sqrt{1 / \rho^{2}+1 / \gamma^{2}}$. Define

$$
\Pi(k)=\prod_{1 \leq j \leq n-1} \min \left\{\gamma^{n-1} M_{x}, 1 /\left|h_{j, j}(k)\right|\right\}^{n-j} .
$$

Lemma 9. For any $k>1$ we have

(i)

$$
\left(\gamma^{n-1} M_{x}\right)^{\left(\begin{array}{l}
n \\
2
\end{array}\right)} \geq \Pi(k) \geq 1
$$

$$
\Pi(k) \geq \tau \Pi(k-1) .
$$

Proof. For the $k$ 's so far, $h_{j, j}(k) \neq 0$ for all $1 \leq j \leq n-1$. Moreover, $M_{x} \geq 1$ and $1 /\left|h_{j, j}(k)\right| \geq 1$ by Lemma 6 . This gives

$$
\min \left\{M_{x}, 1 /\left|h_{j, j}(k)\right|\right\} \geq 1,
$$

for all $1 \leq j \leq n-1$, which implies the right hand inequality of (i). On the other hand, it is always the case that $M_{x} \geq \min \left\{M_{x}, 1 /\left|h_{j, j}(k)\right|\right\}$, which together with the fact that $\left(\begin{array}{l}n \\ 2\end{array}\right)=n-1+\cdots+2+1$ and that $\gamma \geq 1$ gives the left hand inequality of (i).

The proof of part (ii) is more involved. Let $r$ be given by the Step 1 Exchange of PSLQ. Recall the definitions of the two successive diagonal elements $\alpha, \lambda$ and the single off diagonal element $\beta, t=\sqrt{\beta \beta^{\star}+\lambda \lambda^{\star}} /|\alpha|$ in Step 2 (Corner development) of the unitary matrix in terms of $\beta$ and $\lambda$.

Suppose that $r<n-1$. Then only two diagonal elements change. These correspond to the $2 \times 2$ submatrix of $H$

$$
\left(\begin{array}{ll}
\alpha & 0 \\
\beta & \lambda
\end{array}\right)
$$

which after a single iteration becomes

$$
\left(\begin{array}{cc}
\delta & 0 \\
\alpha \beta^{\star} / \delta & -\alpha \lambda / \delta
\end{array}\right)
$$

But $|\delta|=|\alpha| t$, so that the absolute values of the of the $\alpha, \lambda$ diagonal elements are replaced by the absolute values of the $\delta,-\alpha \lambda / \delta$ diagonal elements. All the factors of $\Pi(k)$ are the same except these two, so that

$$
\frac{\Pi(k)}{\Pi(k-1)}=\left(\frac{\min \left\{\gamma^{n-1} M_{x}, 1 /(|\alpha| t)\right\}}{\min \left\{\gamma^{n-1} M_{x}, 1 /|\alpha|\right\}}\right)^{n-r} \cdot\left(\frac{\min \left\{\gamma^{n-1} M_{x}, t /|\lambda|\right\}}{\min \left\{\gamma^{n-1} M_{x}, 1 /|\lambda|\right\}}\right)^{n-r-1} .
$$

Set

$$
A=\gamma^{n-1} M_{x}|\alpha| t \quad \text { and } \quad B=\gamma^{n-1} M_{x}|\lambda|,
$$

so that

$$
\frac{\Pi(k)}{\Pi(k-1)}=\left(\frac{\min \{A, 1\}}{\min \{A, t\}}\right) \cdot\left(\frac{\min \{B, t\}}{\min \{B, 1\}} \cdot \frac{\min \{A, 1\}}{\min \{A, t\}}\right)^{n-r-1} .
$$


We now show that the assumptions for Lemma 7 hold. Note that $1>t$ by the definition of $t$; also, $A \geq B$ since $|\alpha| t \geq|\lambda|$. By Lemma 8 we have $A \geq t \gamma \geq t$. By Lemma 7 we have

$$
\frac{\Pi(k)}{\Pi(k-1)} \geq \frac{\min \{A, 1\}}{\min \{A, t\}} \geq \frac{1}{t} \geq \tau .
$$

Now suppose that $r=n-1$. By Step 3 Reduction, under one iteration the absolute value of the last diagonal element $\alpha$ is less than $|\alpha| \rho$. All the factors of $\Pi(k)$ except the last are the same, so that

$$
\frac{\Pi(k)}{\Pi(k-1)} \leq \frac{\min \left\{\gamma^{n-1} M_{x}, 1 /(|\alpha| \rho)\right\}}{\min \left\{\gamma^{n-1} M_{x}, 1 /|\alpha|\right\}}=\frac{\min \{A, t / \rho\}}{\min \{A, t\}} .
$$

But we always have $\gamma^{n-2} M_{x}|\alpha| \geq 1$, so if $A \geq t / \rho \geq t$, then

$$
\frac{\Pi(k)}{\Pi(k-1)} \geq 1 / \rho \geq \tau \text {. }
$$

By Lemma $8, A \geq t \gamma \geq t$. If $t \leq A \leq t / \rho$, then

$$
\frac{\Pi(k)}{\Pi(k-1)} \geq A / t \geq \gamma \geq \tau .
$$

Thus for $r \leq n-1, \Pi(k) \geq \tau \Pi(k-1)$.

Theorem 2. Assume real or complex numbers, $n \geq 2, \tau>1$, and that $0 \neq x \in \mathbb{K}^{n}$ has $\mathbb{O}(\mathbb{K})$ integer relations. Let $M_{x}$ be the least norm of relations for $x$. Then $P S L Q(\tau)$ will find some integer relation for $x$ in no more than

$$
\left(\begin{array}{l}
n \\
2
\end{array}\right) \frac{\log \left(\gamma^{n-1} M_{x}\right)}{\log \tau}
$$

iterations.

Proof. Suppose we have done $k$ iterations; then, from Lemma 6 and Lemma 7, $\left|h_{j, j}(k)\right| \neq 0$ and not all $\left|h_{j, j}(l)\right|<1 / M_{x}$ for $l<k$. By Lemma $6, \Pi(0) \geq 1$, and by Lemma $7, \Pi(k) \geq \tau^{k}$, so that

$$
\left(\gamma^{n-1} M_{x}\right)^{\left(\begin{array}{c}
n \\
2
\end{array}\right)} \geq \tau^{k} .
$$

Taking natural logarithms of both sides of this inequality gives

$$
\left(\begin{array}{l}
n \\
2
\end{array}\right) \log \left(\gamma^{n-1} M_{x}\right) \geq k \log \tau .
$$

Corollary 2. Let $\mathbb{K}$ be the real numbers $\mathbb{R}$ or the complex numbers $\mathbb{C}$. Fix $n>1$ and assume given a unit n-tuple $x \in \mathbb{K}^{n}$ which has a relation $m_{x} \in \mathbb{O}(\mathbb{K})^{n}$ of least norm $M_{x}$. Then there exists a $\gamma$ such that the algorithm PSLQ $(\tau)$ will construct some $\mathbb{O}(\mathbb{K})^{n}$ relation for $x$ in no more than

$$
2 \cdot\left(\operatorname{dim}_{\mathbb{R}} \mathbb{K}\right) \cdot\left(n^{3}+n^{2} \log M_{x}\right)
$$

iterations.

Proof. Let $\gamma=2$. Then for either $\mathbb{K}, \tau>1$; specifically, $1 / \log \tau<4 \operatorname{dim}_{\mathbb{R}} \mathbb{K}$. $\operatorname{PSLQ}(\tau)$ takes $O(n)$ exact arithmetic operations per iteration, so in this sense finds relations in 'polynomial time' $O\left(n^{4}+n^{3} \log M_{x}\right)$. 


\section{UPPER BOUNDS ON INTEGER RELATIONS}

We compare the relation found by PSLQ to a shortest possible relation.

Lemma 10. Suppose $m$ is the relation found on the $k+1$-st iteration, so that $h_{n-1, n-1}(k+1)=h_{n, n-1}(k)=0$ and $h_{n-1, n-1}(k) \neq 0$. Then

$$
|m|=1 /\left|h_{n-1, n-1}(k)\right| \text {. }
$$

Proof. At this iteration we have developed the matrix $A \in G L(n, \mathbb{O}(\mathbb{K}))$, where the $(n-1)$-st column of $A^{-1}$ by Lemma 5 is $m$ and the vector $A m^{\star}=e_{n-1}$ has as its only non-zero entry a 1 in the $(n-1)$-st position. Since $A P=T D Q$, $Q m^{\star}=D^{-1} T^{\dagger} A m^{\star}$, where $T^{\dagger}$ is the generalized inverse of $T$ and $D$ is a diagonal matrix with last entry $h_{n-1, n-1}(k)$, which is also the last entry of $D^{-1} T^{\dagger} A m^{\star}$. Because $Q$ is unitary, $\left|Q m^{\star}\right|=\left|m^{\star}\right|$.

Theorem 3. Let $M_{x}$ be the smallest possible norm of any relation for $x$. Let $m$ be any relation found by $\operatorname{PSLQ}(\tau)$. For all $\gamma>\sqrt{4 / 3}$ for real vectors and for all $\gamma>\sqrt{2}$ for complex vectors

$$
|m| \leq \gamma^{n-2} M_{x}
$$

Proof. Assume we are at the $k$-th step of PSLQ, where a Step 1 Exchange $r=n-1$ was made with $h_{n-1, n-1}(k) \neq 0$ and $h_{n-1, n-1}(k+1)=0$. Then

$$
\gamma^{n-1}\left|h_{n-1, n-1}(k)\right| \geq \gamma^{j}\left|h_{j, j}(k)\right|
$$

for all $1 \leq j \leq n-2$ by the choice of $r$. Hence, by Theorem 1, Lemma 8 and Lemma 10

$$
M_{x} \geq 1 / \max \left|h_{i, i}(k)\right| \geq \gamma^{2-n} /\left|h_{n-1, n-1}(k)\right|=\gamma^{2-n}|m| .
$$

Comment on Theorem 3. For $n=2$, Theorem 3 proves that any relation $0 \neq m \in$ $\mathbb{O}\left(\mathbb{K}^{2}\right)$ found has norm $|m|=M_{x}$. In other words, $\operatorname{PSLQ}(\tau)$ finds a shortest relation. For real numbers this corresponds to the case of the Euclidean algorithm, $[13$, Book X], [20], [26]. For complex numbers this corresponds to the case of an algorithm in [33].

For $n=3$, let $x=(113,343,311)$. This vector has a shortest relation $m_{x}=$ $(7,-15,14)$ with the shortest norm $\left|m_{x}\right|=M_{x}=21.6794 \ldots$ This can be verified directly; cf. [25], [31], [11]. On the other hand, for $\tau=1.0000 \ldots, \gamma=1.1547 \ldots$, $\operatorname{PSLQ}(\tau)$ in iteration 6 produces the relation $m_{1}=(24,-7,-1)$. Indeed,

$$
M_{x}<\left|m_{1}\right|=25.0199 \ldots \leq \gamma M_{x}=25.0333 \ldots
$$

This relation appears from a zero in the second coordinate of the $x A_{6}^{-1}$ vector. Continuing to iteration 8 gives the relations appearing from the first and second coordinates of the current $x A_{8}^{-1}$ vector, $m_{2}=(-17,-8,15)$ and $m_{3}=(41,1,-16)$ of norms $24.0416 \ldots$ and $44.0227 \ldots$, respectively. The vector $m_{2}$ has smaller but not smallest norm. Continuing to iterations 9 and 10 gives the relations appearing from the first and second coordinates of $x A_{9}^{-1}$ of $m_{4}=(7,-15,14)$ and $m_{2}=(-17,-8,15)$, so a shortest vector $m_{4}$ was eventually found. In iteration 11 the condition $h_{2,2}(11)=0$ appeared for the first time, giving the relation $m_{5}=(-10,-23,29)$ of norm $38.3405 \ldots$. 
This example is instructive in that various choices of the parameter $\tau$ give different outputs. The 'legal' $\tau$ are such that $1<\tau<2$, although the $\operatorname{PSLQ}(\tau)$ sometimes works for 'illegal' $\tau$ outside of this interval. For the 'legal' $\tau=1.1$, iteration 6 yields $m_{1}, 8$ yields $m_{2}, m_{3}, 9$ yields $m_{4}, m_{2}$, and 10 yields $m_{5}$. On the other hand, for $\tau=1.8$, iterations $4,5,6$ all yield only the shortest length relation $m_{4}$. For the 'illegal' $\tau$ below 0.7 and above 2.1 the algorithm cycles indefinitely. The end point $\tau=1.0$ gives essentially the same outputs as $\tau=1.1$. The other end point $\tau=2.0$ yields two new relations, $m_{6}=(1,-91,100)$ and $m_{7}=(0,-311,343)$, of norms $135.2109 \ldots$ and $463.0010 \ldots$, respectively.

\section{Multiple Relations}

A given unit vector $x \in \mathbb{K}^{n}$ may have $0,1,2$, or up to $n-1$ relations. Once a relation has been constructed, one of the coordinates of $x B$ for the appropriate $B \in G L(n, \mathbb{O}(\mathbb{K}))$ will be zero, and the corresponding column of $B$ will be a relation. The remaining $n-1$ coordinates can be used to form a new unit vector in $y \in$ $\mathbb{K}^{n}$. Apply $\operatorname{PSLQ}(\tau)$ to this $y$. Any second relation so found will be integrally independent from the first and can be referred back to the original $x$. In this way as many as $n-1$ integrally independent relations for $x$ can be constructed. We omit here the tangent discussion of using classical lattice reduction techniques to find integer relations; this is the case for the Recognize[ ] function in Mathematica ${ }^{T M}$ which calls the function LatticeReduce[ ], cf. [11], [12], [27]. Lattice reduction there applies typically only to integer relations for integer vectors. Integer relation finding here is directed specifically at integer or Gaussian integer relations for real or complex number vectors.

\section{VARIations OF PSLQ $(\tau)$}

The algorithm $\operatorname{PSLQ}(\tau)$ as stated may be performed for various 'illegal' $\tau$ or 'illegal' $\gamma$, and under these circumstances will find relations for some $x$ vectors. This can happen for $\gamma<\sqrt{4 / 3}$ in the real case, for $\gamma<\sqrt{2}$ in the complex case, and for $\gamma<\infty$ in the quaternion case, so that $\tau<1$ and the conclusions of Theorem 2 or Theorem 3 make no sense or have no apparent content. The reason for this apparent anomaly is that for a specific $n$-tuple $x$ the actual field or division ring constant $\rho$ bound in Lemma 4 is not universal and could depend upon an input vector $x$. Say $\rho_{x}$ gives a bound such as that of Lemma 4 for some special $x$ or collection of them. Then there may be an "illegal" $\gamma$ so that $\tau_{x}=1 / \sqrt{1 / \rho^{2}+1 / \gamma^{2}}>1$. For such $x$ one could expect to see some relation emerge before the number of iterations indicated by Theorem 2 for this $\tau_{x}=\tau$.

On the other hand, it is possible to use the real $\operatorname{PSLQ}(\tau)$ algorithm to find complex and quaternion relations at the expense of doubling and quadrupling the dimension. For example, suppose $z=x+y i+u j+v k$ is a vector in $\mathbb{H}^{n}$ with vector components $x, y, u, v \in \mathbb{R}^{n}$. Suppose the corresponding relation is $m=a+b i+c j+d k$ which is a lattice point in $\mathbb{W}^{n}$ with integral vector components $a, b, c, d \in \mathbb{Z}^{n}$. Then $z m^{\star}=0$ implies four integer relations among the interlaced and suitably sign changed coordinates of $z$. For the first set $\sum_{1 \leq j \leq n}\left(a_{j} x_{j}-b_{j} y_{j}-c_{j} u_{j}-d_{j} v_{j}\right)=0$ and one can apply real $\operatorname{PSLQ}(\tau)$ to the real $4 n$-tuple $\left(\ldots, x_{j}, y_{j}, u_{j}, v_{j}, \ldots\right)$. There are three others which are similar. A relation for $z$ will be in the intersection of the four associated lattices. Alternatively, one can give a $\operatorname{PSLQ}(\tau)$ algorithm along the lines of [23, Section 5. Finding simultaneous integer relations]. 


\section{Computer implementation of $\operatorname{PSLQ}(\tau)$}

The PSLQ $(\tau)$ algorithm can be implemented using ordinary floating point arithmetic on a computer. Using double precision (i.e., 64-bit) arithmetic, relations of two or three digits in size can be recovered for $n$ up to five or so. Beyond this level, precision is quickly exhausted, and recovered relations and norm bounds are meaningless. Thus a serious implementation of PSLQ (or any other integer relation algorithm for real numbers) must employ some form of multiprecision arithmetic. The authors employed the MPFUN multiprecision translator and computation package. The Fortran-77 version of this software is described in [6], and the newer Fortran90 version is described in [7]. A $\mathrm{C}++$ translator that employs these routines is also now available. Alternatively, one may employ the multiprecision facilities of symbolic math software packages, such as Maple, Pari or Mathematica ${ }^{T M}$.

The descriptions presented here of computer implementation of $\operatorname{PSLQ}(\tau)$ are for the case of the real number system. Extensions to the case of the complex and quaternions number systems are straightforward, provided one's multiprecision system supports these datatypes.

One key to an efficient implementation is to utilize a simplified version of Hermite reduction and the associated update. As noted in Lemma 3 above, Hermite reduction can be done more efficiently by a triply nested loop. In fact, the update operations associated with Hermite reduction (updating $x, H, A$ and $B$ ) can also be done in a loop of this form. Further, if these updates are done in this manner, then it is not necessary to compute the $D$ matrix. This simplified scheme is as follows. In the initialization step, Hermite reduction and the subsequent updates are replaced with the following:

For $i$ from 2 to $n$, for $j$ from $i-1$ to 1 (step -1$)$, set $t=\operatorname{nint}\left(h_{i, j} / h_{j, j}\right)$ and replace $x_{j}$ by $x_{j}-t x_{i}$; then for $k$ from 1 to $j$ replace $h_{i, k}$ by $h_{i, k}-t h_{j, k}$; for $k$ from 1 to $n$ replace $a_{i, k}$ by $a_{i, k}-t a_{j, k}$ and replace $b_{k, j}$ by $b(k, j)+t b(k, i)$.

Step 3 is also replaced with this, except $i$ is incremented from $r+1$ to $n$, and $j$ is decremented from $\min \{i-1, r+1\}$ to 1 . Here $r$ denotes the row index selected in Step 1. These more restrictive limits on $i$ and $j$ merely reflect the fact that $t=0$ outside these limits.

Obviously in a computer implementation some care must be taken in testing for zero. This is typically done by checking that the absolute value of the tested value is less than the "epsilon" appropriate for the level of numeric precision being used. Also, a run should be terminated if any entry of the $A$ matrix exceeds the level of numeric precision being used (so that these integer values can no longer be represented exactly).

The level of working precision required for PSLQ is generally only a few digits greater than the accuracy of the input $x$ vector. Along this line, if one wants to recover (or to exclude) relations of size $d$ digits, then the input data must be specified to at least $n d$ digits in order to obtain numerically meaningful results. The significance of a recovered result can be measured by noting the ratio between the multiprecision epsilon and the largest entry of the updated $x$ vector when a relation is recovered. If this ratio is very small, such as $10^{-40}$, then one can be fairly certain that the relation produced by PSLQ is a real relation. But if this ratio is only a few orders of magnitude below unity, then the result is suspect, and higher accuracy in the input data, as well as correspondingly higher working precision, is required. 
The above implementation is satisfactory for most applications. For more demanding applications, a "two-level" implementation is significantly faster. In a twolevel implementation, most operations are performed in ordinary double precision arithmetic, with occasional updates of multiprecision arrays using multiprecision arithmetic. This two-level scheme can be described as follows. Here the prime notation is used to denote double precision approximations to multiple precision values.

To initialize, perform the initialization step as described above using full precision. Then perform an "double precision initialization": (1) set $x^{\prime}=x / \max _{i, j}\left|x_{j}\right|$ and set $H^{\prime}=H$; (2) perform a LQ decomposition on $H^{\prime}$, using double precision arithmetic, setting $H^{\prime}$ to be the lower triangular part; (3) set $A^{\prime}=B^{\prime}=I_{n}$.

PSLQ iterations are then performed as above on the arrays $x^{\prime}, H^{\prime}, A^{\prime}$ and $B^{\prime}$, using double precision arithmetic. Some care must be taken to insure numerical accuracy in these iterations. Obviously these iterations before entries in $A^{\prime}$ grow so large $\left(9 \times 10^{15}\right.$ on IEEE systems $)$ that they cannot be exactly represented as double precision values. In the authors' implementation, double precision iterations are halted when the largest entry of $A^{\prime}$ exceeds $10^{10}$. Tests for zero in these iterations must reflect the accuracy of double precision arithmetic - the authors used an "epsilon" of $10^{-13}$ here. As an additional measure to insure numerical integrity, the authors' code aborts the modified Hermite reduction procedure (and restores arrays to their previous values) if the multiplier $q$ exceeds $10^{7}$.

When the double precision iterations are halted, due either to large entries in $A^{\prime}$, or to a tentative zero in $x^{\prime}$ or $H^{\prime}$, it is necessary to perform a "multiprecision update": (1) replace $A$ by $A^{\prime} A$, replace $B$ by $B B^{\prime}$, replace $H$ by $A^{\prime} H$, and replace $x$ by $x B^{\prime}$; $(2)$ check for zero entries in $x$, using the multiprecision epsilon. If no zeroes are found, then a double precision initialization is performed, followed by more double precision PSLQ iterations.

One detail has been omitted here. In some cases, the entries of the updated $x$ vector have such a large dynamic range (greater than $10^{10}$ in the authors' implementation) that when converted to double precision, additions and subtractions would produce results of questionable reliability. In these cases it is necessary to perform PSLQ iterations on the multiprecision arrays, using multiprecision arithmetic, for a number of iterations until this large dynamic range is eliminated. If this situation is encountered on any iteration other than the very first, a multiprecision LQ decomposition of $H$ must be performed prior to performing these multiprecision iterations (so that the $H$ array contains the same entries as the $H$ array defined in the PSLQ algorithm statement).

The authors' Fortran implementation of PSLQ, together with the required multiprecision arithmetic software, is available by sending electronic mail to dbailey@

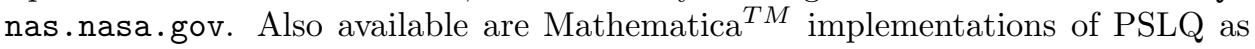
well as a number of other integer relation algorithms for comparison.

\section{Summary of the Literature}

The problem of finding integer relations among sets of rational and real numbers is quite old. When $n=2$ this problem can be solved for rationals by the first Euclidean algorithm in Euclid, Book VII, and for reals by the second Euclidean algorithm given in Euclid, Book X; cf. [26], [11], [37]. Generalizations of this algorithm to higher real dimensions were proposed without proof by many authors, 
including Jacobi [24], Hermite [22], Poincaré [32], Perron [30], Brun [9], [10] and Szekeres [38]. Various counterexamples can be found in [15] and [19].

The first integer relation finding algorithm with proofs for the case of real numbers was discovered in 1977 by Ferguson and Forcade, [14], [15]. These algorithms were shown to be polynomial time in the logarithm of the size of a smallest relation. They were not shown to be polynomial in the dimension. Since then, other related algorithms for finding relations for real vectors have appeared in [8], [16], [17], [18]. For example, [5] reports on a computer implementation of [16]. The sequence including [23] (HJLS), [2] and [1] (PSLQ), [3] (a concise statement of PSLQ), and [35] (a stable variation of HJLS) will be discussed below.

These algorithms all depend upon an orthogonal decomposition of some kind. See [21] for a list of various orthogonalization algorithms and their numerical linear algebra differences. PSLQ is of the QR type. HJLS follows the lattice reduction work of [28], [34], and [36], which is classical Gram-Schmidt type, cf. [31] and [11]. This conceptual difference may explain some of the numerical differences observed between PSLQ and HJLS, cf. [2].

Rigorous proofs that the algorithm under investigation must find a relation if one exists appeared in [14], [8], [15], [16]. All of these proofs gave a linear bound in the logarithm of the size of a relation, but were not known to be polynomial in the dimension. [8] and [16] had unsatisfactory proofs in the sense that they were shown to be at worst exponential in the dimension rather than polynomial in the dimension. This unsatisfactory state of affairs was resolved affirmatively with the proofs that appeared in [23] for the 'small integer relation algorithm'. We will refer to this 'small integer relation algorithm' as HJLS, as stated in [23, Section $3]$ as a reflection of that in [8, Section 3]. In fact, this proof in [23] was the first appearance in the literature of a 'polynomial time' bound for a relation finding algorithm, polynomial in both dimension and logarithm of relation size.

This important progress was made when [23] combined two independent streams of research, [14], [8], [15], [16], [18] and [28], [29], [34], [35], [11]. Inspired by the polynomial result of [23], but not the details, the first author of this paper formulated what he thought was a new algorithm [2], [1] and gave a polynomial proof. This proof was independent of that of [23], a different analysis, but flawed by giving a slightly higher degree polynomial in the dimension than the polynomial proof given in [23]. This algorithm in [2], [1] was called PSLQ and had the advantage of the adjustable parameter $\gamma$ or $\tau$. Applications and implementation of this earlier version of $\operatorname{PSLQ}(\tau)$ were described in [3], [7], [4]. These implementations showed that the parameters were a helpful feature of the algorithm. The bound on iterations for HJLS proven in [23] was $O\left(n^{3}+n^{2} \log _{2} M_{x}\right)$; this is consonant with the bound proven in this paper for $\operatorname{PSLQ}(\sqrt{2})$. The subsequent paper [35] included parameters as well as addressing a certain issue of stability.

As a specific example, consider the triple $x=(11,27,31)$. We list the sequence of $A^{-1}$ matrices for each algorithm. A relation if found will be constructed as a column of one of these $A^{-1}$ matrices.

For PSLQ(1.1547) the successive iterations $k=0,1,2,3,4$, yield the five $A^{-1}$ matrices

$$
\left(\begin{array}{ccc}
1 & 0 & 0 \\
0 & 1 & 0 \\
0 & -1 & 1
\end{array}\right),\left(\begin{array}{ccc}
1 & 0 & 0 \\
3 & 8 & 1 \\
-3 & -7 & -1
\end{array}\right),\left(\begin{array}{ccc}
-2 & 1 & 0 \\
2 & 3 & 1 \\
-1 & -3 & -1
\end{array}\right)
$$




$$
\left(\begin{array}{ccc}
3 & -2 & 0 \\
1 & 2 & 1 \\
-2 & -1 & -1
\end{array}\right),\left(\begin{array}{ccc}
-1 & -8 & -2 \\
5 & 9 & 2 \\
-4 & -5 & -1
\end{array}\right)
$$

Note that PSLQ has constructed two relations appearing as the first and second columns of the last matrix, iteration $k=4$.

For HJLS the successive iterations $k=0,1,2,3,4,5,6$ yield the seven $A^{-1}$ matrices

$$
\begin{gathered}
\left(\begin{array}{lll}
1 & 0 & 0 \\
0 & 1 & 0 \\
0 & 0 & 1
\end{array}\right),\left(\begin{array}{ccc}
1 & 0 & 0 \\
0 & 0 & 1 \\
0 & 1 & -1
\end{array}\right),\left(\begin{array}{ccc}
0 & 1 & 0 \\
0 & 0 & 1 \\
1 & 0 & -1
\end{array}\right),\left(\begin{array}{ccc}
1 & -2 & 0 \\
0 & 0 & 1 \\
0 & 1 & -1
\end{array}\right), \\
\left(\begin{array}{ccc}
1 & 0 & -2 \\
0 & 1 & 2 \\
0 & -1 & -1
\end{array}\right),\left(\begin{array}{ccc}
0 & 1 & -2 \\
1 & 3 & 2 \\
-1 & -3 & -1
\end{array}\right),\left(\begin{array}{ccc}
0 & -2 & -1 \\
1 & 2 & 5 \\
-1 & -1 & -4
\end{array}\right) .
\end{gathered}
$$

Note that only one relation is found; it appears in the last column of the last matrix, iteration $k=6$. The authors of [34] claimed that HJLS is a special case of PSLQ $(\tau)$ for $\gamma=\sqrt{2}$ or equivalently $\tau=\sqrt{4 / 3}$. The example just given shows that this claim cannot be true.

The significance of the parameter was revealed clearly in the extensive tables appearing in [2]. In [2] the parameter was $\gamma$, which is equivalent to giving $\tau=$ $1 / \sqrt{\rho^{2}+1 / \gamma^{2}}$. The choice of the parameter $\tau$ has precision consequences: depending upon the choice of parameter, a numerical precision much higher than that of the input real vector must be used to obtain a reliable result. For example, the algebraic number

$$
\alpha=3^{1 / 4}-2^{1 / 4}=\sqrt{\sqrt{3}}-\sqrt{\sqrt{2}}
$$

satisfies a polynomial of degree 16 with coefficients

$$
(1,0,0,0,-3860,0,0,0,-666,0,0,0,-20,0,0,0,1) .
$$

The algorithm $\operatorname{PSLQ}(\tau)$ for $\tau=1.000006145$ or $\gamma=1.1547005384$, applied to the vector $\left(1, \alpha, \alpha^{2}, \cdots, \alpha^{n-1}\right)$, with $n=17$, finds these coefficients with a working precision of 75 decimal digits. We have shown with the $n=3$ example above that HJLS is not PSLQ $(\sqrt{4 / 3})$. Again, we see that HJLS requires a working precision of more than 10,000 decimal digits to find this $n=17$ relation. Comparative run times are not particularly relevant here but are also correspondingly higher for HJLS - see Table 2 of [2].

For a slightly different $\tau=\sqrt{4 / 3}=1.154700538 \ldots, \operatorname{PSLQ}(\tau)$ requires 85 decimal digits, 10 digits more than for $\gamma=1.1547005384$. Generally the closer $\tau$ is to 1 the less precision seems to be required. This observed phenomenon appears to have nothing to do with any question of numerical stability.

The various algorithms in the literature stand independently of their published proofs; their published proofs may not reveal their actual properties clearly. Though the proofs were exponential, the algorithms stated in [14], and in [15], and again in [16] were parametric. The parameter $b$ in [14], [15] satisfies $1<b<2$, whereas in [16] the parameter $\gamma$ is emphasized. The algorithm in $[8$, Sect. $\mathrm{x}]$ seems closest to $\operatorname{PSLQ}(\sqrt{4 / 3})$ with the $\tau$ parameter set by $\gamma=\sqrt{2}$. This parameter choice appears in [8, Sect. $\mathrm{x}]$ without the [28] setting and reappears in [23] as the "small 
integer relation algorithm", which we call HJLS, rewritten in the [28] language and accompanied by a 'polynomial time' proof for the first time.

Bergman discussed the complex case of finding Gaussian integer relations for complex vectors in $[8$, Sect. 5: Variants]. Bergman also gave an algorithm for the simultaneous real vector case in $[8$, Sect. 7]. Following Bergman, the paper defining HJLS for simultaneous real vectors, [23, cf. Sect. 5]; implicitly includes the complex and quaternion vector case as well. As an alternate approach, inspired by [37], in this paper we have extended the base field of PSLQ $(\tau)$ to these division rings and introduced unitary matrices into the algorithm directly. The proof given here of polynomial number of iterations covers the real and complex cases, but fails for quaternions. However, the quaternion version of $\operatorname{PSLQ}(\tau)$ performs reasonably well experimentally in finding Hamiltonian integer relations for quaternion vectors. This was explained in Section 8.

\section{Open questions}

1) Is there a relation finding algorithm that finds a shortest relation in a polynomial (in the dimension) number of iterations?

2) What are the best choices for the parameter $\tau$ or $\gamma$ relative to the number of iterations, time, and precision requirements of PSLQ?

\section{ACKnowledgments}

The authors thank (in alphabetical order) Peter Borwein, M. Euchner, Rod Forcade, Jeff Lagarias, Alyson Reeves, Robert Riley, M. L. Robinson, Carsten Rössner, Claus Schnorr, and Francis Sullivan for their motivating comments about PSLQ. Specifically, we thank Alyson Reeves for her lucid rewriting of the proof of Lemma 5, Rodney Forcade for counterexamples, and the referee for clarifications.

\section{REFERENCES}

1. Steve Arno and Helaman Ferguson, A new polynomial time algorithm for finding relations among real numbers, Supercomputing Research Center Tech Report SRC-93-093 (March 1993), 1-13.

2. D. H. Bailey and H. R. P. Ferguson, A polynomial time, numerically stable integer relation algorithm, SRC Technical Report SRC-TR-92-066; RNR Technical Report RNR-91-032 (16 December 1991; 14 July 1992), 1-14.

3. D. H. Bailey, J. Borwein, and R. Girgensohn, Experimental evaluation of Euler sums, Experimental Mathematics 3 (October 1994), 17 - 30. MR 96e:11168

4. D. H. Bailey, P. Borwein, and S. Plouffe, On the rapid computation of various polylogarithmic constants, Mathematics of Computation 66 (218) (April 1997), 903 - 913. CMP 97:06

5. D. H. Bailey, Numerical results on the transcendence of constants involving $\pi$, $e$, and Euler's constant, Mathematics of Computation 50 (181) (January 1988), 275 - 281. MR 88m:11056

6. D. H. Bailey, Multiprecision translation and execution of Fortran programs, ACM Transactions on Mathematical Software 19 (3) (1993), 288 - 319.

7. D. H. Bailey, A Fortran-90 based multiprecision system, ACM Transactions on Mathematical Software 21 (4) (1995), 379 - 387..

8. G. Bergman, Notes on Ferguson and Forcade's generalized Euclidean algorithm, University of California at Berkeley, unpublished notes, Nov. 1980.

9. V. Brun, En generalisatiken av kjedebroøken, I, II, Norske Videnskapsselskapets Skrifter I. Matematisk Naturvidenskapelig Klasse 6 (1919, 1920), 1-29, 1-24.

10. V. Brun, Algorithmes euclidiens pour trois et quatre nombres, Treizième Congrès des mathematiciens Scandinaves, tenu a Helsinki 18-23 août 1957, Mercators Trycheri, Helsinki, 1958, pp. 46-64. MR 22:2597 
11. H. Cohen, A Course in Computational Algebraic Number Theory, Graduate Texts in Mathematics 138, Springer-Verlag, Berlin Heidelberg New York, 1993. MR 94i:11105

12. M. J. Coster, A. Joux, B. A. LaMacchia, A. M. Odlyzko, C. P. Schnorr, J. Stern, Improved low-density subset sum algorithms, Computational Complexity 2 (1992), no. 2, 111-128. MR 94e: 11141

13. Euclid, translated from the text of Heiberg with introduction and commentary by Sir Thomas L. Heath, The Thirteen Books of Euclid's Elements, Second Edition, revised with additions, unabridged, Volumes I, II, III, Dover Publications, Inc., New York, 1956. MR 17:814b

14. H. R. P. Ferguson and R. W. Forcade, Generalization of the Euclidean algorithm for real numbers to all dimensions higher than two, Bulletin (New Series) of the American Mathematical Society 1 (1979), 912 - 914. MR 80i:11039

15. H. R. P. Ferguson and R. W. Forcade, Multidimensional Euclidean algorithms, (Crelle's) Journal für die reine und angewandte Mathematik 334 (1982), 171 - 181. MR 84d:10015

16. Helaman Ferguson, A short proof of the existence of vector Euclidean algorithms, Proceedings of the American Mathematical Society 97 (1) (May 1986), 8 - 10. MR 87k:11080

17. Helaman Ferguson, A noninductive $G L(n, Z)$ algorithm that constructs integral linear relations for $n$ Z-linearly dependent real numbers, Journal of Algorithms (8) (1987), 131 - 145. MR 88h:11096

18. Helaman Ferguson, PSOS: A new integral relation finding algorithm involving partial sums of squares and no square roots, Abstracts of papers presented to the American Mathematical Society 9 (56; 88T-11-75) (March 1988), 214.

19. Rodney W. Forcade, Brun's algorithm, unpublished manuscript (November 1981), $1-27$.

20. David Fowler, Ratio in early Greek mathematics, Bulletin (New Series) of the American Mathematical Society 1 (6) (November 1979), 807 - 846. MR 82c:01008

21. G. H. Golub and C. F. Van Loan, Matrix Computations, 2nd Edition, The Johns Hopkins University Press, Baltimore, Maryland, 1990. MR 90d:65055

22. C. Hermite, Extraits de lettres de M. Ch. Hermite à M. Jacobi sur differénts objets de la théorie de nombres, (Crelle's) Journal für die Reine und Angewandte Mathematik $(3,4)$ (1850), $261-315$.

23. J. Hastad, B. Just, J. C. Lagarias, and C. P. Schnorr, Polynomial time algorithms for finding integer relations among real numbers, SIAM Journal of Computing 18 (1989), 859 - 881. MR 90g:11171

24. C. G. J. Jacobi, Allgemeine Theorie der Kettenbruchahnlichen Algorithmen, in welchen jede Zahl aus drei vorhergehenden gebildet wird (Aus den hinterlassenen Papieren von C. G. J. Jacobi mitgetheilt durch Herrn E. Heine.), Journal für die Reine und Angewandte Mathematik 69 (1) (1868), $29-64$.

25. R. Kannan, Lattices, basis reduction, and the shortest vector problem, Theory of Algorithms (Pécs, 1984), Colloquia Mathematica Societatis János Bolyai, vol. 44, North-Holland, Amsterdam, 1985, pp. 283-311. MR 87m:90087

26. D. E. Knuth, The Art of Computer Programming, Vol. 2 Seminumerical Algorithms, Second Edition, Addison-Wesley, Reading, MA, 1981. MR 83i:68003

27. J. C. Lagarias, H. W. Lenstra Jr., and C. P. Schnorr, Korkin-Zolotarev bases and successive minima of a lattice and its reciprocal lattice, Combinatorica 10 (4) (1990), 333 - 348. MR 92a: 11075

28. A. K. Lenstra, H. W. Lenstra Jr., and L. Lovasz, Factoring polynomials with rational coeffcients, Math. Ann. 261 (1982), 515 - 534. MR 84a:12002

29. Laszlo Lovasz and Herbert E. Scarf, The generalized basis reduction algorithm, Mathematics of Operations Research 17 (3) (August 1992), 751 - 764. MR 93h:52023

30. O. Perron, Grundlagen für eine Theorie des Jacobischen Kettenbruchalgorithmus, Math. Ann. (64) (1907), $1-76$

31. M. Pohst and H. Zassenhaus, Algorithmic Algebraic Number Theory, Chapter 3: Methods from the Geometry of Numbers, Encyclopedia of Mathematics and its Applications, Cambridge University Press, New York, 1989. MR 92b:11074

32. H. Poincaré, Sur une généralisation des fractions continues, Comptes Rendus Acad. Sci. Paris 99 (1884), 1014 - 1016.

33. Asmus L. Schmidt, Diophantine approximation of complex numbers, Acta Mathematica 134 (1975), $1-85$. MR 54:10160 
34. C. Schnorr and M. Euchner, Lattice basis reduction: improved practical algorithms and solving subset sum problems, Fundamentals of Computation Theory (Gosen, 1991), Lecture Notes in Computer Science, vol. 529, Springer-Verlag, Berlin, Heidelberg, New York, 1991, pp. 68-85; also published in Mathematical Programming Series A 66 (2) (1994), 181-199. MR 92g:68007; MR 95j:90064

35. C. Rössner and C. P. Schnorr, A stable integer relation algorithm, FB Mathematik/ Informatik Universität Frankfurt TR-94-016 (1994), 1 - 11.

36. C. P. Schnorr, A more efficient algorithm for lattice basis reduction, Journal of Algorithms 9 (1988), 47 - 62. MR 89h:11086

37. G. Shimura, Fractional and trigonometric expressions for matrices, The American Mathematical Monthly 101 (8) (October 1994), 744 - 758. MR 96e:15053

38. G. Szekeres, Multidimensional continued fractions, Ann. Univ. Sci. Budapest Eötvös Sect. Math. 13 (1970), 113 - 140. MR 47:1763

Center for Computing Sciences, 17100 Science Drive, Bowie, MD 20715-4300

E-mail address: helamanf@super.org

E-mail address: arno@super.org

Lawrence Berkeley Lab, Mail Stop 50B-2239, Berkeley, CA 94720

E-mail address: dhb@nersc.gov 